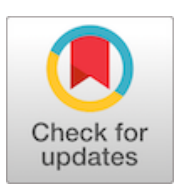

\title{
De la puerta de urgencias al quirófano: revelando los minutos de oro en trauma cardíaco penetrante
}

\author{
From the ER door to the operating room: Revealing the golden minutes \\ in penetrating cardiac trauma
}

José Eduardo Agamez-Fuentes ${ }^{1} \mathbb{D}$, Daniel Ernesto Mejía ${ }^{1} \mathbb{D}$, Sandra Sepúlveda ${ }^{1} \mathbb{D}$, Brayan Muñoz-Caicedo ${ }^{2} \mathbb{D}$, David Mejía-Toro ${ }^{3} \mathbb{D}$, Carlos Hernando Morales-Uribe $^{4}(\mathbb{D}$

1 Médico, residente de Cirugía general, Universidad de Antioquia, Medellín, Colombia.

2 Médico, residente de radiología, Universidad de Antioquia, Medellín, Colombia.

3 Médico, especialista en Cirugía general y Cirugía de trauma y emergencias, Hospital Universitario San Vicente Fundación y Hospital Pablo Tobón Uribe; profesor de Cirugía general, Universidad de Antioquia, Medellín, Colombia.

4 Médico, especialista en Cirugía general y Cirugía de trauma y emergencias; profesor de Cirugía general, Universidad de Antioquia, Medellín, Colombia.

\section{Resumen}

Introducción. El manejo de la herida cardiaca penetrante es un reto dado que requiere un rápido manejo quirúrgico para evitar que su desenlace sea fatal. Múltiples factores pronósticos han sido descritos, sin embargo, no ha sido documentada la relación entre el tiempo de llegada a quirófano y el uso de pledgets con la mortalidad.

Métodos. Se realizó un estudio observacional retrospectivo de corte transversal, desde el año 2011 hasta el año 2018 , en un hospital universitario de la ciudad de Medellín. Se evaluaron los registros de los pacientes con herida cardiaca penetrante confirmada y se realizó análisis univariado, bivariado y multivariado, así como curvas de supervivencia.

Resultados. Los pacientes inestables o con taponamiento cardiaco que llegan al quirófano después del minuto 4 de haber ingresado a urgencias tienen cuatro veces más posibilidades de morir que los que llegan a quirófano antes (RR 4,1 IC ${ }_{95 \%}$ 1,43-12,07). El uso de pledgets en el reparo de la herida cardiaca, corresponde a un factor protector para los pacientes, con un OR ajustado de $2,5\left(\mathrm{IC}_{95 \%}\right.$ 1,124-5,641). El tipo de traumatismo, la arritmia intraoperatoria y el índice de choque al ingreso también fueron factores pronósticos.

Discusión. Se documenta el efecto del tiempo de llegada a quirófano sobre la mortalidad, lo cual permitirá en un futuro generar cambios en el manejo de estos pacientes en función de estos tiempos. La evidencia encontrada sugiere mejores desenlaces con el uso rutinario de pledgets.

Palabras clave: heridas penetrantes; lesiones cardíacas; urgencias médicas; procedimientos quirúrgicos; tiempo de tratamiento; mortalidad.

Fecha de recibido: 15/09/2020 - Fecha de aceptación: 15/11/2020 - Fecha de publicación en línea: 19/04/06 Correspondencia: José Eduardo Agamez-Fuentes, Calle 36 A \# 58 - 55, Bello, Colombia. Teléfono (+57) 3188664905 Correo electrónico: joseeduardoagamez@gmail.com

Citar como: Agamez-Fuentes JE, Mejía DE, Sepúlveda S, Muñoz-Caicedo B, Mejía-Toro D, Morales-Uribe CH. De la puerta de urgencias al quirófano: revelando los minutos de oro en trauma cardíaco penetrante. Rev Colomb Cir. 2021;36:427-37. https://doi.org/10.30944/20117582.841

Este es un artículo de acceso abierto bajo una Licencia Creative Commons - BY-NC-ND https://creativecommons.org/licenses/by-ncnd/4.0/deed.es 


\begin{abstract}
Introduction. The management of penetrating cardiac injury is challenging since it requires rapid surgical management to avoid a fatal outcome. Multiple prognostic factors have been described, however, the relationship between the time of arrival to the operating room and the use of pledgets with mortality has not been documented.
\end{abstract}

Methods. A cross-sectional retrospective observational study was conducted from 2011 to 2018 in a university hospital in the city of Medellín. Records of patients with confirmed penetrating cardiac injury were evaluated, and univariate, bivariate, and multivariate analyzes were performed, as well as survival curves.

Results. Unstable patients or patients with cardiac tamponade who arrive to the operating room after 4 minutes after being admitted to the emergency room are four times more likely to die than those who arrive to the operating room earlier (RR 4.195\% CI 1.43-12.07). The use of pledgets in the repair of the cardiac wound corresponds to a protective factor for patients, with an adjusted OR of 2.5 (95\% CI 1.124-5.641). The type of trauma, intraoperative arrhythmia and the shock index on admission were also prognostic factors.

Discussion. The effect of the time of arrival to the operating room on mortality is documented, which will allow in the future to generate changes in the management of these patients based on these times. The evidence found suggests better outcomes with the routine use of pledgets.

Keywords: penetrating wounds; heart injuries; emergencies; surgical procedures; time-to-treatment; mortality.

\section{Introducción}

Desde 1996, la violencia urbana es catalogada por la Organización Mundial de la Salud (OMS) como un problema de salud pública ${ }^{1}$. El trauma originado por el conflicto armado en Colombia ha generado una gran carga social, económica, política y humanitaria. Para el año 2018, Colombia tuvo una tasa de mortalidad por homicidios de 24 por cien mil personas y en el departamento de Antioquia dicha tasa fue de 34 por cien mil personas, cifra que duplica la mortalidad por accidentes de tránsito. De esta manera, en el 2018 se perdieron a causa de los homicidios 401.420 años de vida entre los hombres de 15 a 55 años y cerca de 93.984 años de vida saludable en lesiones no fatales en el mismo segmento poblacional ${ }^{2}$. En otros países del mundo también es clara la gran carga para los sistemas de salud como consecuencia de los traumatismos por violencia, tanto torácicos como cardíacos ${ }^{3-6}$.

En Colombia no se cuenta con estadísticas claras respecto al trauma cardiaco; sin embargo, en experiencias previamente documentadas en el Hospital Universitario San Vicente Fundación (HUSVF) en Medellín, se ha reportado una frecuencia de trauma cardiaco cercana a las 50 heri- das por año ${ }^{7-9}$. Dada la alta incidencia de heridas cardiacas penetrantes en la ciudad de Medellín y el HUSVF, la optimización y estandarización de los protocolos de manejo de estos pacientes es un objetivo primordial, en búsqueda de mejorar los desenlaces.

No hay duda de que el lapso de tiempo disponible para la atención de un paciente con herida cardiaca penetrante es corto. De hecho, es aceptado que entre más rápida sea la intervención, los desenlaces serán mejores; sin embargo, esto no ha sido cuantificado de una manera sistemática y objetiva. Lo mismo ocurre respecto al rol de los pledgets en la sutura de heridas cardiacas, pues hasta el momento las recomendaciones para su uso corresponden a opiniones de expertos.

Adicionando estas dos variables al estudio, se pretende explicar el comportamiento de la mortalidad de los pacientes con herida cardiaca penetrante y generar una nueva evidencia que permita mejorar los protocolos de manejo.

\section{Métodos}

Se realizó un estudio observacional retrospectivo, de corte transversal, en el Hospital Universitario San Vicente Fundación, de la ciudad de Medellín, 
Colombia, a partir de una muestra recolectada desde el año 2011 hasta el año 2018, donde se evaluaron los pacientes con herida cardiaca penetrante demostrada en la exploración quirúrgica, incluidas las heridas pericárdicas o epicardicas. Los pacientes incluidos ingresaron al servicio de urgencias por lo menos con signos de vida (pulso carotideo, ventilación o esfuerzo ventilatorio, respuesta pupilar, movimientos, actividad cardíaca detectada en cardioscopio o en ultrasonido).

A partir de las historias clínicas se registraron en una base de datos las siguientes variables: número de historia clínica, fecha de ingreso, edad, genero, signos vitales de ingreso (frecuencia respiratoria, frecuencia cardiaca, presión arterial sistólica, escala de coma de Glasgow), mecanismo de trauma, localización de la herida, herida cardiaca compleja, condición de ingreso, tiempo de llegada a quirófano, método diagnóstico, arritmia intraoperatoria, uso de pledgets, estado de egreso.

El tiempo de llegada al quirófano fue definido de acuerdo al registro quirúrgico, en donde una auxiliar de la institución llena la hora exacta de ingreso a quirófano y se resta a esta hora la hora de recepción del paciente en el sistema, la cual es consignada en el formato de ingreso del paciente por la secretaria del servicio de admisiones, proceso que en los casos de emergencia toma menos de 1 minuto.

Respecto al índice de choque (IC), en los pacientes en cuyo caso no se sensaba presión arterial sistólica, se asignó un valor de $34 \mathrm{mmHg}$, dado que, de acuerdo con su ficha técnica, el límite mínimo de detección de tensión arterial no invasiva de los monitores de signos vitales disponibles en la institución, es de $35 \mathrm{mmHg}$.

Se analizaron los datos en el programa estadístico IBMSPSS versión 24. Se llevó a cabo un análisis univariado con los resúmenes de estadísticos descriptivos; para las variables cualitativas se determinaron las frecuencias absolutas y porcentuales, para las variables cuantitativas se analizó la distribución de sus datos mediante la prueba de Kolmogorov - Smirnov, se calcularon sus medidas de tendencia central y de dispersión. Posteriormente se realizó un análisis bivariado entre la variable dependiente mortalidad y las demás variables; según la naturaleza de las variables se realizaron pruebas $\mathrm{T}$ de student, prueba $U$ de Mann-Whitney o Chi cuadrado de Pearson. Para el análisis multivariado se realizó una regresión logística binaria mediante el método ENTER y con el fin de ajustar las medidas de fuerza de asociación (OR) encontradas en el análisis bivariado, ingresaron al modelo las variables de mayor interés clínico y las que en el análisis bivariado presentaron un valor de $\mathrm{p}<0,25$ (criterio de Hosmer-Lemeshow).

\section{Resultados}

Características sociodemográficas, de ingreso y de la atención del paciente

Se atendieron 326 pacientes con herida cardiaca penetrante desde enero de 2011 hasta diciembre de 2018. El $94 \%$ de los pacientes fueron hombres, con un promedio de edad de 31 años (tabla 1). Al ingreso, el promedio de frecuencia respiratoria fue de 14 respiraciones por minuto, de frecuencia cardiaca, 88 latidos por minuto y de presión arterial sistólica, $69 \mathrm{mmHg}$ (tabla 2). El índice de choque promedio fue de 1,53 y el RTS (Revised trauma score) promedio fue de 4,8 .

Tabla 1. Descripción de las características sociodemográficas, de ingreso y de la atención de los pacientes con herida cardiaca penetrante.

\begin{tabular}{llcc}
\hline Variable & Categoría & Frecuencia & $\%$ \\
\hline \multirow{2}{*}{ Género } & Femenino & 19 & 5,8 \\
& Masculino & 307 & 94,2 \\
\hline \multirow{2}{*}{ Mecanismo } & Arma cortopunzante & 304 & 93,3 \\
del trauma & Arma de fuego & 20 & 6,1 \\
& Artefacto explosivo & 2 & 0,6 \\
\hline \multirow{4}{*}{ Condición } & Estable & 70 & 21,5 \\
& "In extremis" & 68 & 20,9 \\
& Inestabilidad hemodinámica & 158 & 48,5 \\
& Paro cardíaco & 12 & 3,7 \\
& Taponamiento cardiaco & 18 & 5,5 \\
\hline \multirow{3}{*}{ Diagnóstico } & Ecografía subxifoidea & 203 & 62,3 \\
& Laparoscopia & 118 & 36,2 \\
& Ventana pericárdica & 1 & 0,3 \\
\hline
\end{tabular}


Tabla 2. Descripción de las variables cuantitativas sociodemográficas, de ingreso y de la atención de los pacientes con herida cardiaca penetrante

\begin{tabular}{|c|c|c|c|c|c|c|c|}
\hline Variable & Media & Mediana & D.E & RIC & Min & Max & $\begin{array}{c}\text { Kolmogorov - } \\
\text { Smirnov ** }\end{array}$ \\
\hline Edad & 31 & 28 & 11 & 15 & 11 & 68 & 0,000 \\
\hline $\begin{array}{l}\text { Frecuencia } \\
\text { respiratoria }\end{array}$ & 14,4 & 14 & 6 & 8 & 0 & 30 & 0,000 \\
\hline Frecuencia cardiaca & 88,7 & 93 & 32 & 40 & 0 & 150 & 0,000 \\
\hline Presión sistólica & 69 & 70 & 36 & 34 & 0 & 160 & 0,000 \\
\hline Glasgow & 10 & 13 & 4,7 & 9 & 3 & 15 & 0,000 \\
\hline $\begin{array}{l}\text { Tiempo para llegar } \\
\text { al quirófano }\end{array}$ & 31 & 10 & 245 & 12 & 1 & 4320 & 0,000 \\
\hline Días en UCl & 3,4 & 2 & 6,2 & 3 & 0 & 44 & 0,000 \\
\hline Días no UCl & 4,8 & 3 & 5,6 & 4 & 0 & 44 & 0,000 \\
\hline $\begin{array}{l}\text { Tiempo de cirugía } \\
\text { (minutos) }\end{array}$ & 81 & 70 & 50 & 39 & 11 & 405 & 0,000 \\
\hline RTS & 4,8 & 5,4 & 2,5 & 4,5 & 0,0 & 7,8 & 0,000 \\
\hline Índice de choque & 1,5 & 1,3 & 0,8 & 0,9 & 0,0 & 5,0 & 0,001 \\
\hline
\end{tabular}

*D.E: Desviación estándar; RIC: rango intercuartílico, Min: mínimo, Max: Máximo.

${ }^{* *}$ Valor < 0,00: no normal

El principal mecanismo de trauma fue por arma cortopunzante, que se presentó en 304 pacientes $(93,3 \%)$, mientras 20 pacientes $(6,1 \%)$ sufrieron herida por proyectil de arma de fuego y 2 pacientes $(0,6 \%)$ a causa de esquirlas asociadas a una explosión. El ventrículo derecho fue el sitio anatómico más comprometido, con un total de 195 pacientes (59,8\%), seguido del ventrículo izquierdo, que se documentó en 72 pacientes $(22,1 \%)$.

La principal presentación clínica fue la inestabilidad hemodinámica, en 158 pacientes (48,5\%); 70 $(21,5 \%)$ pacientes ingresaron estables, $68(20,9 \%)$ ingresaron "In extremis", 18 pacientes (5,5\%) tenían taponamiento cardiaco clínicamente evidente y 12 pacientes $(3,7 \%)$ ingresaron en paro. Se tomó la decisión de llevar al paciente a cirugía solo por su estado clínico al ingreso (sin realización de imagen diagnostica) en 203 pacientes $(62,3 \%)$ y después de realizar ecografía subxifoidea en 118 pacientes (36,2\%).

La mediana de tiempo entre el ingreso al servicio de urgencias y el ingreso a quirófano fue de 10 minutos y en promedio una cirugía tomó 81 minutos en su realización. Durante la intervención quirúrgica, 53 pacientes (16,3\%) tuvieron fibrilación o taquicardia ventriculares sin pulso y 19 pacientes (5.8\%) presentaron actividad eléctrica sin pulso o asistolia.

La clasificación de la herida cardiaca fue predominantemente grado IV, con un total de 229 pacientes $(70,2 \%)$, seguido de grado $\mathrm{V}$, en 85 pacientes $(26,1 \%)$; las heridas cardiacas grado III, II y I fueron una minoría (tabla 3). En 202 pacientes (62\%) se usó pledgets para la cardiorrafia. En el posoperatorio los pacientes tuvieron una media de estancia hospitalaria en UCI de 3 días y en sala general de 5 días. Murieron 52 (16\%) de 326 pacientes de esta serie.

\section{Factores de mortalidad}

Al tomar como referencia los pacientes que al ingreso estaban estables hemodinámicamente, en el análisis bivariado de mortalidad se encontró que los pacientes inestables hemodinámicamente te-

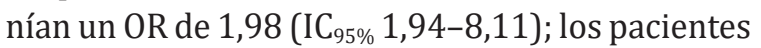

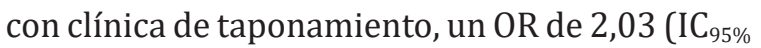
0,35-12,27); los pacientes "In extremis", un OR de $7,89\left(\mathrm{IC}_{95 \%} 2,55-24,43\right) \mathrm{y}$, los pacientes en paro, un

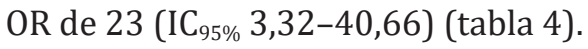


Tabla 3. Variables relacionadas con el tratamiento y la evolución de los pacientes.

\begin{tabular}{lccc}
\hline Variable & Categoría & Frecuencia & $\%$ \\
\hline & 2 o más cámaras & 14 & 4,3 \\
& Aurícula derecha & 26 & 8,0 \\
Localización de la herida & Aurícula izquierda & 9 & 2,8 \\
cardiaca & Epicárdica & 3 & 0,9 \\
& Pericárdica & 7 & 2,1 \\
& Ventrículo derecho & 195 & 59,8 \\
& Ventrículo izquierdo & 72 & 22,1 \\
\hline \multirow{4}{*}{ Clasificación de la herida } & Grado I & 1 & 0,3 \\
cardiaca & Grado II & 2 & 0,6 \\
& Grado III & 8 & 2,5 \\
& Grado IV & 229 & 70.2 \\
\multirow{2}{*}{ Herida cardiaca compleja } & Grado V & 85 & 26,1 \\
\hline \multirow{2}{*}{ Uso de pledgets } & Herida coronaria & 18 & 5,5 \\
& Herida septal & 3 & 0,9 \\
\multirow{2}{*}{ Arritmia intraoperatoria } & Herida valvular & 1 & 0,3 \\
& No & 304 & 93,3 \\
\hline \multirow{2}{*}{ Estado al egreso } & No & 123 & 37,7 \\
& Si & 202 & 62,0 \\
\hline
\end{tabular}

Tabla 4. Factores de ingreso y de la atención asociados a mortalidad.

\begin{tabular}{|c|c|c|c|c|c|c|c|c|}
\hline \multirow{2}{*}{ Variable } & & \multicolumn{2}{|c|}{ Muerto } & \multicolumn{2}{|c|}{ Vivo } & \multirow{2}{*}{ OR } & \multirow{2}{*}{ p } & \multirow{2}{*}{ IC95\% } \\
\hline & & $\mathbf{n}$ & $\%$ & $\mathbf{n}$ & $\%$ & & & \\
\hline \multirow{3}{*}{ Mecanismo de trauma } & Arma cortopunzante & 40 & 13,2 & 264 & 86,8 & 1 & - & \\
\hline & Arma de fuego & 11 & 55,0 & 9 & 45,0 & 8,06 & 0,000 & $3,15-20,68$ \\
\hline & Artefacto explosivo & 1 & 50,0 & 1 & 50,0 & 6,6 & 0,12 & $0,4-107,6$ \\
\hline \multirow{7}{*}{$\begin{array}{l}\text { Localización de la herida } \\
\text { cardiaca * }\end{array}$} & 2 o más cámaras & 3 & 21,4 & 11 & 78,6 & 1,6 & 0,5 & - \\
\hline & Aurícula derecha & 5 & 19,2 & 21 & 80,8 & 1,4 & - & - \\
\hline & Aurícula izquierda & 0 & 0,0 & 9 & 100,0 & - & - & - \\
\hline & Epicárdica & 0 & 0,0 & 3 & 100,0 & - & - & - \\
\hline & Pericárdica & 2 & 28,6 & 5 & 71,4 & 2,3 & - & - \\
\hline & Ventrículo derecho & 28 & 14,4 & 167 & 85,6 & 1 & - & - \\
\hline & Ventrículo izquierdo & 14 & 19,4 & 58 & 80,6 & 1,43 & 0,5 & 0,71 \\
\hline \multirow{5}{*}{ Condición de ingreso } & Estable & 4 & 5,7 & 66 & 94,3 & 1 & - & - \\
\hline & "In extremis" & 22 & 32,4 & 46 & 67,6 & 7,89 & 0,00 & $2,55-24,43$ \\
\hline & Inestabilidad hemodinámica & 17 & 10,8 & 141 & 89,2 & 1,98 & 0,00 & $1,94-8,11$ \\
\hline & Paro & 7 & 58,3 & 5 & 41,7 & 23 & 0,00 & $3,32-40,66$ \\
\hline & Taponamiento cardiaco & 2 & 11,1 & 16 & 88,9 & 2,03 & 0,07 & $0,35-12,27$ \\
\hline \multirow{4}{*}{ Diagnóstico \# } & Clínico & 48 & 23,6 & 155 & 76,4 & 8,82 & 0,00 & $3,09-25,18$ \\
\hline & Ecografía subxifoidea & 4 & 3,4 & 114 & 96,6 & 1 & - & - \\
\hline & Laparoscopia & 0 & 0,0 & 1 & 100,0 & - & - & - \\
\hline & Ventana pericárdica & 0 & 0,0 & 3 & 100,0 & - & - & - \\
\hline \multirow{4}{*}{ Arritmia intraoperatoria } & Ritmo desfibrilable & 25 & 47,2 & 28 & 52,8 & 12,4 & 0,00 & $6,0-25,8$ \\
\hline & Ritmo no desfibrilable & 10 & 52,6 & 9 & 47,4 & 15,4 & 0,00 & $2,08-11,93$ \\
\hline & No & 17 & 6,7 & 237 & 93,3 & 1 & - & - \\
\hline & $\begin{array}{c}\text { Desfibrilable Vs no } \\
\text { desfibrilable }\end{array}$ & & & & & 1,24 & 0,68 & - \\
\hline \multirow{2}{*}{ Pledgets } & No & 31 & 25,2 & 92 & 74,8 & 2,9 & 0,00 & $1,58-5,34$ \\
\hline & $\mathrm{Si}$ & 21 & 10,4 & 181 & 89,6 & 1 & - & - \\
\hline \multirow{5}{*}{$\begin{array}{l}\text { Clasificación de la herida } \\
\text { cardiaca + }\end{array}$} & Grado I & 1 & 100 & 0 & 0,0 & - & - & - \\
\hline & Grado II & 0 & 0,0 & 2 & 100 & - & - & - \\
\hline & Grado III & 1 & 12,5 & 7 & 87 & 1 & - & - \\
\hline & Grado IV & 32 & 14 & 197 & 86 & 1,13 & 0,9 & $0,14-9,55$ \\
\hline & Grado V & 18 & 21,2 & 67 & 78,8 & 1,88 & 0,56 & $0,22-16,29$ \\
\hline
\end{tabular}

*Se compara el riesgo de mortalidad entre herida en ventrículo derecho y herida en ventrículo izquierdo por conglomerar la mayoría de los pacientes y ser la asociación de mayor importancia clínica.

\# Se compara el riesgo de mortalidad entre el diagnóstico por ecografía subxifoidea y el diagnóstico por condición clínica por conglomerar la mayoría de los pacientes.

+ Se compara el riesgo de mortalidad entre la herida grado IV y grado $V$ por conglomerar la mayor cantidad de pacientes y ser la de mayor importancia clínica. 
Los pacientes que presentaron arritmia intraoperatoria tenían un OR de 12,4 (IC ${ }_{95 \%} 3,32-$ $40,66)$ para ritmos desfibrilables y un OR de 15,4 (IC $\left.{ }_{95 \%} 2,08-11,93\right)$ para ritmos no desfibrilables, al comparar con los pacientes sin arritmia intraoperatoria.

Los pacientes con heridas en ventrículo izquierdo tuvieron un OR para mortalidad de 1,43 $\left(\mathrm{IC}_{95 \%}\right.$ 0,71-2,92) comparando con las heridas en ventrículo derecho, y los pacientes en los cuales no se usó pledgets tuvieron un OR de 2,9 ( IC $_{95 \%}$ 1,58-5,34) en comparación con los pacientes en los que se usaron pledgets.

Respecto al índice de choque, se encontró que la mediana para los pacientes que fallecieron fue significativamente superior en comparación con los sobrevivientes (diferencia de medianas de $0,39, \mathrm{p}<0,01$ ). El RTS tuvo un comportamiento similar, con un valor menor para la mediana en los pacientes que murieron (diferencia de medianas de 2,37, $\mathrm{p}<0,01$ ) (tabla 5).

\section{Análisis multivariado}

Para el análisis multivariado se ingresaron al modelo las variables de mayor importancia identificadas en el análisis bivariado: mecanismo del trauma, RTS, índice de choque, condición al ingreso, arritmia intraoperatoria y uso de pledgets. En el modelo multivariado los determinantes de mortalidad que alcanzaron la significancia estadística fueron: lesión por arma de fuego, con un OR ajustado de 40,7 (IC ${ }_{95 \%}$ 1,97-841,74); arritmia intraoperatoria, con un OR ajustado de 15,47 ( $\left.\mathrm{IC}_{95 \%} 4,24-56,43\right)$; índice de choque, con un OR ajustado $2,6(\mathrm{p}=0.05)$, y no uso de pledgets, con

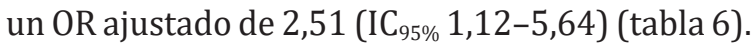

\section{Tiempo de llegada a quirófano}

Respecto a los tiempos de llegada a quirófano, la mediana fue de 10 minutos. Se realizaron figuras de supervivencia, para observar el comportamiento de la sobrevida de los pacientes respecto al tiempo que tardaron en ser llevados a manejo quirúrgico. La figura 1 demuestra el comportamiento de la población total y se observa que la sobrevida al minuto 1, 2, 3, 4 y 5 es de 94,4 \%, 94,4 \%, 92,8 \%, 91,5 \% y 89,7 \% respectivamente, confiriendo así unos riesgos relativos (RR) de muerte de 2,9, 2,9, $2,3,2$ y 1,7 para los pacientes que fueron operados

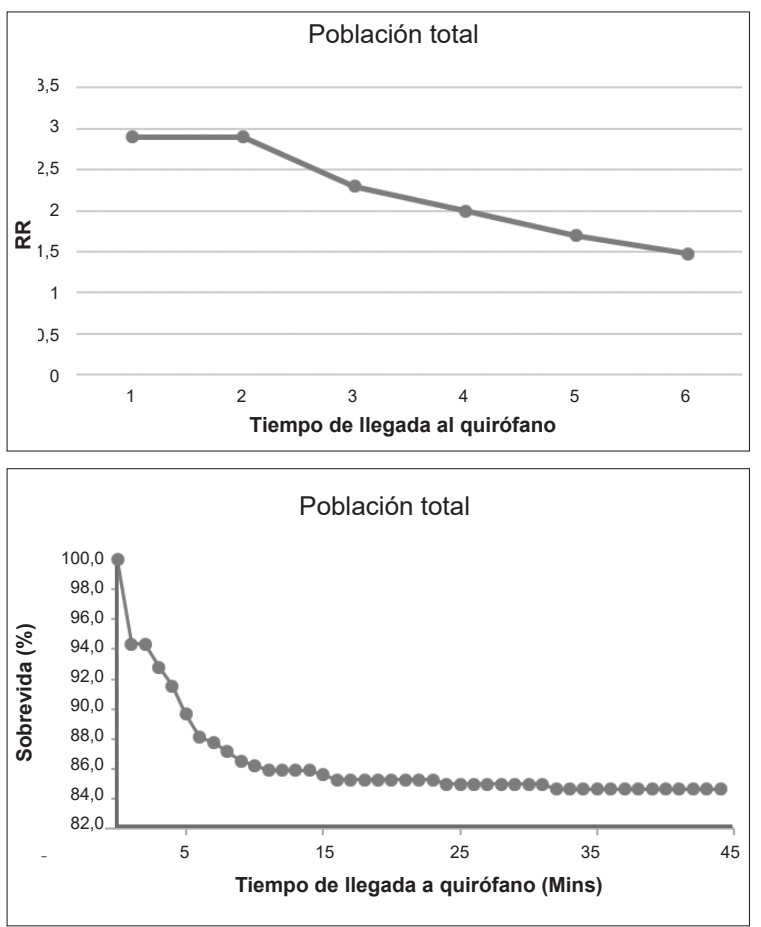

Figura 1. Sobrevida de la población total según el tiempo de llegada al quirófano.

Tabla 5. Diferencia en los índices de trauma entre los pacientes que murieron y sobrevivieron.

\begin{tabular}{lcccccc}
\hline \multicolumn{7}{c}{ Estado al egreso } \\
\hline & Media & Mediana & Media & Mediana & $\begin{array}{c}\text { Diferencia de } \\
\text { medianas }\end{array}$ & $\mathbf{p}$ \\
\hline RTS* $^{*}$ & 3,704 & 3,656 & 5,062 & 5,967 & 2,37 & 0,00 \\
Índice de choque & 1,91 & 1,64 & 1,46 & 1,25 & 0,39 & 0,00 \\
\hline
\end{tabular}

${ }^{*}$ RTS: revised trauma score 
Tabla 6. Regresión logística con variables de mayor riesgo de mortalidad y mayor interés clínico.

\begin{tabular}{|c|c|c|c|c|}
\hline & \multirow[b]{2}{*}{ p } & \multirow[b]{2}{*}{$\begin{array}{c}\text { OR } \\
\text { ajustado }\end{array}$} & \multicolumn{2}{|c|}{$\mathrm{IC}_{95 \%}$} \\
\hline & & & $\begin{array}{l}\text { Límite } \\
\text { inferior }\end{array}$ & $\begin{array}{l}\text { Límite } \\
\text { superior }\end{array}$ \\
\hline $\begin{array}{l}\text { Condición clínica } \\
\text { de ingreso }\end{array}$ & 0,149 & & & \\
\hline Inestable & 0,295 & 3,157 & 0,367 & 27,169 \\
\hline "In extremis" & 0,498 & 0,534 & 0,087 & 3,283 \\
\hline Paro cardíaco & 0,937 & 0,932 & 0,162 & 5,372 \\
\hline $\begin{array}{l}\text { Taponamiento } \\
\text { cardíaco }\end{array}$ & 0,319 & 0,341 & 0,041 & 2,821 \\
\hline RTS & 0,919 & 1,049 & 0,419 & 2,624 \\
\hline Índice de choque & 0,020 & 2,975 & 1,184 & 7,473 \\
\hline Arma de fuego & 0,016 & 40,718 & 1,970 & 841,748 \\
\hline $\begin{array}{l}\text { Arritmia } \\
\text { intraoperatoria }\end{array}$ & 0,000 & 15,477 & 4,245 & 56,432 \\
\hline No uso de pledgets & 0,025 & 2,518 & 1,124 & 5,641 \\
\hline
\end{tabular}

después de cada uno de esos intervalos de tiempo, respectivamente. Por encima del minuto 5 de tardanza en tiempo de llegada al quirófano, el riesgo relativo pierde significancia estadística.

Se realizó el mismo análisis para el subgrupo de pacientes inestables y taponados, donde la sobrevida era de $97,4 \%, 97,4 \%, 97,4 \%$ y $96,1 \%$, con unos RR de 4,16, 4,16, 4,16 y 2,8, para los pacientes que fueron llevados a quirófano después en el minuto 1, 2, 3 y 4 . Después del minuto 4 el RR pierde significancia estadística, tal como se observa en la figura 2.

Para las pacientes "In extremis" no se encontró diferencia significativa en los RR para ninguno de los minutos de llegada a quirófano (figura 3 ).

\section{Discusión}

Han pasado más de 20 años desde que se realizaron los primeros estudios locales acerca del trauma cardiaco penetrante, y conforme pasa el tiempo se modifican las condiciones sociodemográficas, los métodos diagnósticos, terapéuticos, quirúrgicos y de seguimiento, lo cual repercute directamente en los desenlaces de los pacientes. Con
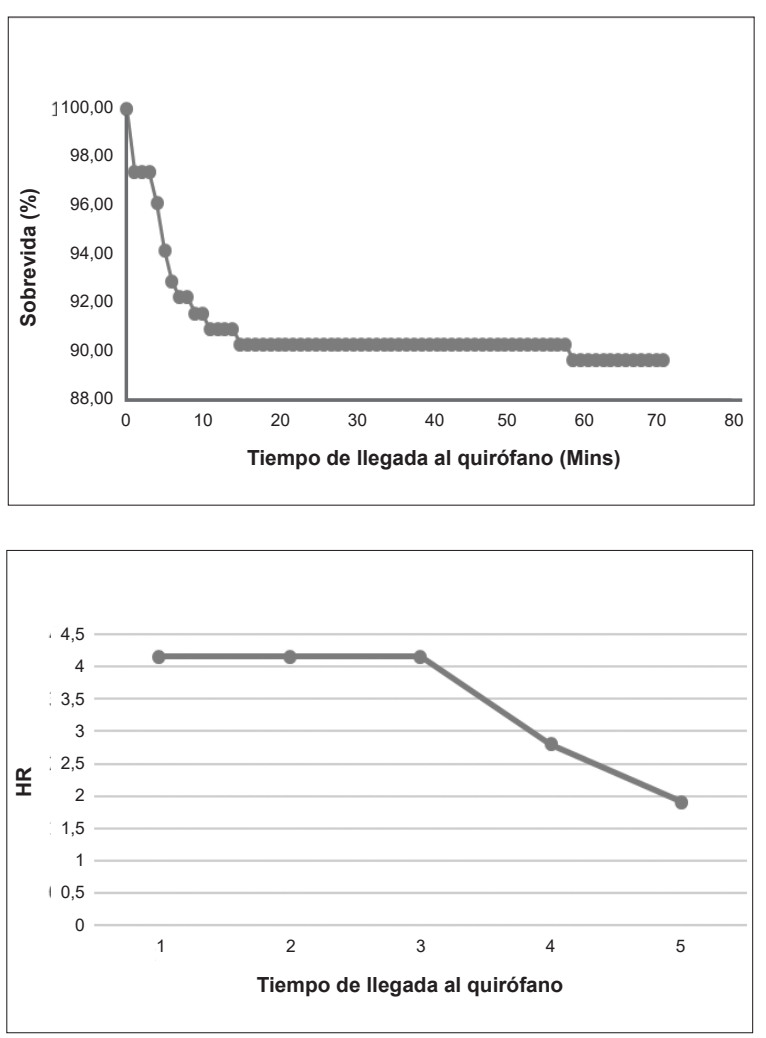

Figura 2. Sobrevida en pacientes inestables o con taponamiento cardíaco según tiempo de llegada al quirófano.

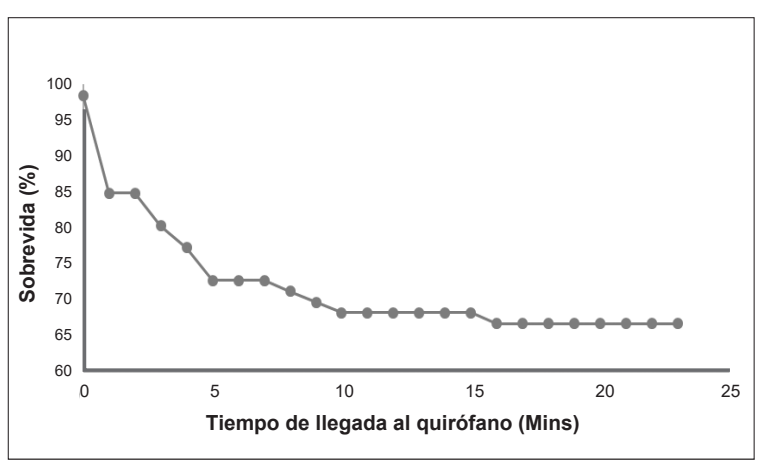

Figura 3. Sobrevida en pacientes "In extremis" según el tiempo de llegada al quirófano.

el presente estudio se buscó actualizar la experiencia ya documentada y aportar nueva evidencia al evaluar dos variables que hasta el momento solo obedecían al concepto de expertos: el tiempo de llegada a quirófano y el uso de pledgets. 


\section{Características sociodemográficas y mecanismos de lesión}

La serie de pacientes documentada en el presente estudio es muy similar a las experiencias locales reportadas anteriormente, los lesionados corresponden en su mayoría de pacientes jóvenes (31 años en promedio), masculinos (94\%), heridos por arma cortopunzante (93\%), que ingresan inestables hemodinámicamente ( $48 \%$ ) y son llevados a cirugía basándose en la presentación clínica de ingreso (62\%). Una vez en cirugía, se identifica con mayor frecuencia una herida de ventrículo derecho (60\%).

Llama la atención el bajo número de pacientes con taponamiento cardiaco, respecto a otras series, donde se ha documentado que en general un tercio de los pacientes llegan en estado de choque, un tercio taponados y un tercio estables. Es posible que dentro del grupo de pacientes inestables o "In extremis" se encontraran pacientes taponados y que dicho estado clínico no haya sido notado y/o anotado por el médico de urgencias en la historia clínica al momento del ingreso. En nuestra serie, la mortalidad global fue del $16 \%$, un valor muy similar a la de otras series locales ${ }^{8-10}$.

Respecto a otros reportes internacionales, persiste una diferencia notable en el mecanismo del trauma. Es conocido que, en Estados Unidos, durante los años 80 y 90, la tasa de heridas cardiacas penetrantes por arma cortopunzante duplicaba las heridas por proyectil de arma de fuego, sin embargo, en las últimas dos décadas hubo un cambio en la frecuencia del mecanismo, y en la actualidad, las lesiones por proyectil de arma de fuego se presentan en un $63 \%$ de los pacientes, mientras los traumas por arma blanca solo ocurren en un $30 \%$ de los $\operatorname{casos}^{11,12}$. Estas diferencias en los mecanismos condicionan las tasas de mortalidad. En países desarrollados la tasa de mortalidad de las heridas cardiacas por proyectil de arma de fuego es de 45-90 \% y por arma blanca del 19-24 \% ${ }^{12-14}$, en comparación con nuestra serie, donde se observa una mortalidad del $55 \%$ y el 13,2\% respectivamente para cada tipo de mecanismo.
En publicaciones internacionales, la localización más frecuente de la herida fue en el ventrículo derecho (60\%), al igual que la presente serie. Sin embargo, las tasas de lesión del ventrículo izquierdo y las heridas multicamerales sí presentan una diferencia amplia, siendo menores en nuestra serie: $37 \%$ vs $22 \%$ y $18 \%$ vs $4,3 \%$, lo que posiblemente es explicado por la menor frecuencia de heridas por proyectil de arma de fuego en el presente estudio ${ }^{14}$.

\section{Parámetros fisiológicos de ingreso y gravedad de las lesiones}

Los pacientes que fallecieron tuvieron una diferencia estadísticamente significativa en los signos vitales de ingreso con respecto a los pacientes que sobrevivieron. Esto se visualiza de mejor manera en el cálculo del RTS y el índice de choque, donde se aprecia la diferencia marcada en el estado fisiológico de los pacientes que sobreviven en comparación con los que fallecen, que alcanza la significancia estadística.

Respecto a la gravedad de las lesiones según la clasificación de la American Association for the Surgery of Trauma (AAST), solo disponemos de un reporte colombiano, de Isaza y colaboradores ${ }^{10}$, donde documentaron la distribución y mortalidad de heridas según su grado. Lo primero que se encuentra es una diferencia ostensible en la distribución de las heridas, dado que en el estudio de Izasa la frecuencia de heridas cardiacas grado II y III fue cercana al $46 \%$ mientras en la presente serie fue aproximadamente del 3,5\%, una distribución muy similar a la cifra de $6 \%$, reportada previamente para la misma institución ${ }^{8}$. La tasa de mortalidad de acuerdo a la clasificación de las heridas es similar al estudio de Izasa, donde documentaron mortalidad de 2,5 \%, 12,5 $\%, 20 \%$ y $25,4 \%$ para las heridas cardiacas grado II, III, IV y V respectivamente, mientras en la presente serie se reportaron cifras de $0 \%, 12,5$ $\%, 14 \%$ y $21,2 \%$.

\section{Características de la atención}

En el HUSVF la decisión de realizar toracotomía se ha definido la mayoría de las veces según el 
juicio clínico, y no mediante ayudas diagnósticas, ya que se considera que esta decisión cobra especial importancia, dado que el tiempo perdido en llevar el paciente a un procedimiento diagnóstico puede afectar la sobrevida ${ }^{7,8}$. Esto se ve reflejado en la serie actual, donde el $62 \%$ de los pacientes fueron llevados al quirófano basados en el estado clínico de ingreso, sin la realización de ecografía subxifoidea ni FAST (Focused Abdominal Sonography in Trauma).

Esta decisión, aunada a que los quirófanos de la institución se encuentran a menos de 40 metros del ingreso de urgencias, ha permitido acortar significativamente los tiempos de ingreso a quirófano de los pacientes; de hecho, en la práctica clínica en el HUSVF, hay pacientes que en menos de 1 minuto se encuentran en el quirófano, lo cual se documentó en 2 pacientes en el presente estudio. Por las razones anteriores, en el HUSVF no se realiza con frecuencia toracotomía de emergencia fuera de quirófano, como sí sucede en otros países, donde realizan toracotomías en el departamento de emergencia hasta en el $25 \%$ de los pacientes ${ }^{14}$.

Respecto al manejo preoperatorio, al realizar las curvas de supervivencia, se destaca el hallazgo de la relación entre el tiempo de llegada al quirófano y la sobrevida. De la misma manera se realizó una comparación de riesgos relativos para los pacientes operados antes del minuto $2,3,4,5 \mathrm{y}$ 6 , encontrándose que la probabilidad de muerte es casi tres veces más alta si el paciente se opera después del minuto 2 (RR 2,9, IC $\mathrm{I}_{95 \%} 1,64-5,25$ ), y después del minuto 5 se pierde la significancia estadística del riesgo relativo.

Cuando se realiza el mismo análisis para el subgrupo de pacientes con taponamiento cardiaco o inestables, se encuentra un efecto mucho más marcado del tiempo de llegada al quirófano, con un RR que se mueve entre 4,16 (IC I5\% $_{1,43-12,07)}$ y 2,8 (IC $\left.{ }_{95 \%} 1,14-6,91\right)$, para los pacientes llevados al quirófano después del minuto 3 y el minuto 4 respectivamente, y después del minuto 4 se pierde la significancia estadística del RR para este subgrupo de pacientes. Este fenómeno, está en relación directa con la caída abrupta de la mortalidad para los pacientes inestables después del minuto 3 , tal como se observa en su curva de supervivencia en la gráfica 2 .

Para los pacientes que ingresan "In extremis", se encontró que la mortalidad disminuye con cada minuto que se tarde en llevar el paciente al quirófano, sin embargo, los RR no alcanzaron significancia estadística, lo cual puede estar en relación con un estado de choque descompensado, en un punto de no retorno, en donde a pesar de la instauración de rápidas medidas de control del sangrado, algunos pacientes progresan a la muerte de manera inevitable.

Respecto al manejo quirúrgico, se evaluó en esta serie el uso de pledgets para la sutura de la herida cardiaca, recomendación que hasta el momento sigue con un nivel de evidencia de "concepto de expertos". Las publicaciones son divergentes, mientras algunos autores recomiendan el uso rutinario de pledgets, otros solo recomiendan su uso en el caso de maceración del tejido o desgarro del miocardio durante la sutura (15-17). En el 37,7 \% de nuestros pacientes no se usó pledgets, y al realizar el análisis bivariado se estimó una mortalidad del 25,2 \% para esos pacientes, en comparación con una mortalidad del 10,4\% para los pacientes en los que sí se usó pledgets, con un OR de 2,9 (IC $95 \%$ 1,58-5,34).

Al realizar el análisis multivariado incluyendo las variables mecanismo del trauma, RTS, índice de choque, condición al ingreso, arritmia intraoperatoria y uso de pledgets, también se encontró una asociación estadísticamente significativa, con un OR ajustado de 2,51 (IC $\left._{95 \%} 1,12-5,64\right)$, lo cual confirma que el uso de pledgets corresponde a una variable independiente predictora de muerte.

En el intraoperatorio, también cabe destacar que los pacientes que presentaron arritmia cardíaca intraoperatoria tuvieron mayor probabilidad de muerte $y$, a pesar que hay una tendencia a mayor mortalidad en los pacientes con ritmos no desfibrilables, al comparar la tasa de mortalidad con los pacientes con ritmos desfibrilables, no se alcanzó una diferencia estadísticamente significativa. 


\section{Conclusiones}

Este estudio permitió realizar un abordaje más exhaustivo de los factores pronósticos en los pacientes con herida cardiaca penetrante en el entorno local. Después del análisis de los resultados, encontramos que estos son concordantes con la literatura disponible, mostrando factores predictores de muerte como el mecanismo del trauma, la arritmia cardiaca intraoperatoria y el índice de choque.

Adicionalmente, se generó nueva evidencia científica, que sugiere que los pacientes que ingresan con signos clínicos de taponamiento cardíaco o con inestabilidad hemodinámica deben ser llevados a quirófano antes del minuto cuatro, ya que de lo contrario la tasa de mortalidad se triplica. En el HUSVF es factible la consecución de este objetivo puesto que el quirófano se encuentra a menos de 40 metros del ingreso de urgencias; sin embargo, en instituciones donde el transporte del paciente desde el servicio de urgencias a cirugía tome más de 3 minutos, la recomendación a partir de la evidencia generada sería realizar toracotomía en el servicio de urgencias.

Por último, a partir de nuestros datos, sugerimos también el uso rutinario de pledgets para la sutura de heridas cardiacas, en especial si son grado IV o V, como ocurrió en la mayoría de la población de nuestro estudio.

\section{Cumplimiento de normas éticas}

Consentimiento informado: Este estudio es una revisión de historias clínicas retrospectiva, y como tal, no hay necesidad de un consentimiento informado. El Comité de Ética Institucional aprobó el diseño y la metodología del estudio.

Conflicto de interés: Ninguno declarado por los autores.

Fuentes de financiación: Autofinanciado por los autores.

\section{Contribución de los autores:}

- Concepción y diseño del estudio: José Eduardo AgamezFuentes, Daniel Ernesto Mejía, Sandra Sepúlveda, Brayan Muñoz-Caicedo, David Mejía-Toro, Carlos Hernando Morales-Uribe

- Adquisición de datos: José Eduardo Agamez-Fuentes, Daniel Ernesto Mejía, Sandra Sepúlveda, Brayan Muñoz-
Caicedo, David Mejía-Toro, Carlos Hernando MoralesUribe

- Análisis e interpretación de datos: José Eduardo AgamezFuentes, Daniel Ernesto Mejía, Sandra Sepúlveda, Brayan Muñoz-Caicedo, David Mejía-Toro, Carlos Hernando Morales-Uribe.

- Redacción del manuscrito: José Eduardo AgamezFuentes, Daniel Ernesto Mejía, Sandra Sepúlveda, Brayan Muñoz-Caicedo.

- Revisión crítica: David Mejía-Toro, Carlos Hernando Morales-Uribe.

\section{Referencias}

1. Krug EG, Dahlberg LL, Mercy JA, Zwi AB, Lozano R. World report on violence and health. Fecha de consulta: 25 de noviembre de 2018. Disponible en: http://apps.who.int/iris/bitstream/handle/10665/ 42495/9241545615_eng.pdf;jsessionid=75B96D8F584B4A529277E12EEAC8675A? sequence $=1$

2. Instituto Nacional de Medicina Legal y Ciencias Forenses. Forensis 2018 datos para la vida. Fecha de consulta: 12 de octubre de 2019. Disponible en: https:// cnnespanol.cnn.com/wp-content/uploads/2019/06/ forensis-2018.pdf

3. Lecky FE, Bouamra O, Woodford M, Alexandrescu R, O’Brien SJ. Epidemiology of polytrauma. In: Pape H-C, Peitzman AB, Schwab CW, Giannoudis PV, editors. Damage control management in the polytrauma patient. New York: Springer; 2010. p. 13-24.4.

4. Ziegler DW, Agarwal NN. The morbidity and mortality of rib fractures. J Trauma. 1994; 37:975-979. https://doi.org/10.1097/00005373-199412000-00018

5. Beshay M, Mertzlufft F, Kottkamp HW, Reymond M, Schmid RA, Branscheid D, Vordemvenne T. Analysis of risk factors in thoracic trauma patients with a comparison of a modern trauma centre: a mono-centre study. World J Emerg Surg. 2020;15. https://doi.org/10.1186/s13017-020-00324-1

6. Mandal AK, Sanusi M. Penetrating chest wounds: 24 years experience. World J Surg. 2001; 25:1145-9. https://doi.org/10.1007/BF03215862

7. Castano W, Morales CH, Senior JM, Benjumea WY, Sanchez J. Relationship of echocardiographic and coronary angiographic findings in patients with acute myocardial infarction secondary to penetrating cardiac trauma. J Trauma Acute Care Surg. 2012;73:111-6. https://doi.org/10.1097/TA.0b013e318256a0d8

8. Duque HA, Florez LE, Moreno A, Jurado H, Jaramillo CJ, Restrepo MC. Penetrating cardiac trauma: follow-up study including electrocardiography, echocardiography, and functional test. World J Surg. 1999;23:1254-7. https://doi.org/10.1007/s002689900658 
9. Villegas-Lanau MI, Morales-Uribe $\mathrm{CH}$, Rosero-Cerón E, Benítez-España G, Cano-Restrepo F, Fernández IM, et al. Trauma cardíaco penetrante: factores pronósticos. Rev Colomb Cir. 2007;22:148-56.

10. Isaza-Restrepo A, Bolívar-Sáenz DJ, Tarazona-Lara M, Tovar JR. Penetrating cardiac trauma: analysis of 240 cases from a hospital in Bogota, Colombia. World J Emerg Surg. 2017;12:26-33. https://doi.org/10.1186/s13017-017-0138-1

11. Bellister SA, Dennis BM, Guillamondegui OD. Blunt and penetrating cardiac trauma. Surg Clin N Am. 2017;97:1065-76.

https://doi.org/10.1016/j.suc.2017.06.012

12. Asensio JA, Ogun OA, Petrone P, Perez-Alonso AJ, Wagner M, Bertellotti, R, et al. Penetrating cardiac injuries: predictive model for outcomes based on 2016 patients from the National Trauma Data Bank. Eur J Trauma Emerg Surg. 2018;44:835-41. https://doi.org/10.1007/s00068-017-0806-6
13. Thourani VH, Feliciano DV, Cooper WA, Brady KM, Adams AB, Rozicky GS, Symbas PN. Penetrating cardiac trauma at an urban trauma center: a 22-year perspective. Am Surg. 1999;65:811-8.

14. Morse BC, Mina MJ, Carr JS, Jhunjhunwala R, Dente CJ, Zink JU, et al. Penetrating cardiac injuries: A 36-year perspective at an urban, Level I trauma center. J Trauma Acute Care Surg. 2016;81:623-31. https://doi.org/10.1097/TA.0000000000001165

15. Evans J, Gray LA, Jr Rayner A, Fulton RL. Principles for the management of penetrating cardiac wounds. Ann Surg. 1979;189:777-84.

https://doi.org/10.1097/00000658-197906000-00015

16. Moore EE, Feliciano DV, Mattox KL, editors. Trauma. $8^{\text {th }}$ ed. New York: McGrawHill Medical; 2017.

17. Jacobs LM, Luk SS. The cardiovascular system. Advanced Trauma Operative Management. 2nd edition. Connecticut: Ciné-Med Publishing, Inc; 2010. p. 239-300. 